\title{
Parent Artery Occlusion for Unruptured Cerebral Aneurysms: Results of the Japanese Registry of Neuroendovascular Therapy 3
}

\author{
Hidehisa NisHI, ${ }^{1}$ Akira IsHII, ${ }^{1}$ Tetsu SATOw, ${ }^{2}$ Koji IIHARA, ${ }^{3}$ \\ Nobuyuki SAKAI, ${ }^{4,5}$ and Japanese Registry of \\ Neuroendovascular Therapy (JR-NET) investigators \\ ${ }^{1}$ Department of Neurosurgery, Graduate School of Medicine, Kyoto University, \\ Kyoto, Kyoto, Japan; \\ ${ }^{2}$ Department of Neurosurgery, National Cerebral and Cardiovascular Center, \\ Suita, Osaka, Japan; \\ ${ }^{3}$ Department of Neurosurgery, Graduate School of Medical Sciences, \\ Kyushu University Fukuoka, Fukuoka, Japan; \\ ${ }^{4}$ Department of Neuroendovascular Therapy, Institute of Biomedical Research \\ and Innovation, Kobe, Hyogo, Japan; \\ ${ }^{5}$ Department of Neurosurgery, Kobe City Medical Center General Hospital, Kobe, Hyogo, Japan
}

\begin{abstract}
Although the current standard treatment for unruptured aneurysms comprises surgical clipping or endovascular coiling, these techniques are not suitable for some cases, such as large, giant, and fusiform aneurysms. Endovascular parent artery occlusion (PAO), which includes internal trapping and proximal occlusion, is a well-established alternative treatment for such cases. Here, we retrospectively reviewed PAO cases from the Japanese Registry of Neuroendovascular Therapy 3, a nation-wide survey of all neuroendovascular therapy cases between 2010 and 2014. This dataset included 274 procedures with a mean patient age of 57.1 years and $55.4 \%$ female patients. For the treatment strategy, internal trapping was selected in 213 aneurysm cases $(77.7 \%)$ and proximal occlusion in 61 aneurysm cases $(22.2 \%)$. Most of the procedures were successfully completed (272/274: 99.2\%). Immediately after treatment, angiographical complete occlusion was achieved in 248 cases $(90.5 \%)$. Although the feasibility of this technique was excellent, there were 60 periprocedural complications ( $21.8 \%$ ), including 48 ischemic complications ( $17.5 \%)$, seven hemorrhagic complications $(2.5 \%)$. Overall, morbidity and mortality at 30 days postoperative were $5.8 \%$ and $0.7 \%$, respectively. Among the pretreatment variables, a patient age of 70 and older was associated with ischemic complications [odds ratio (OR); $2.34,95 \%$ confidence interval (CI); 1.02-5.25; $P=0.04$ ] and a small aneurysm size $(<5 \mathrm{~mm})$ was associated with hemorrhagic complications (OR; 9.85, 95\% CI; 1.07-221.0; $P=0.04$ ) by multivariate analysis. In conclusion, PAO for unruptured cerebral aneurysms is feasible, but is associated with a complication rate of approximately $20 \%$. Various alternative treatment options should be carefully considered with deconstructive strategies.
\end{abstract}

Key words: parent artery occlusion, unruptured cerebral aneurysm, Japanese Registry of Neuroendovascular Therapy

\section{Introduction}

After a multi-center randomized trial revealed the feasibility and safety of endovascular coiling for the

Received August 31, 2018; Accepted October 22, 2018

Copyright $\subset 2019$ by The Japan Neurosurgical Society This work is licensed under a Creative Commons AttributionNonCommercial-NoDerivatives International License. treatment of intracranial aneurysms, this technique has become popular worldwide. ${ }^{1)}$ However, the treatment of large, giant, wide-necked, and fusiform aneurysms remains technically challenging. ${ }^{2)}$

Despite advancements in techniques and devices for treating such aneurysms, such as bioactive coils, hydrogel coils, the double catheter technique, compliant balloons, and neck-bridging stents, clinical studies report that the recanalization rate for the 
aneurysm coiling is still high, i.e., more than $10 \%$ with those difficult aneurysms. ${ }^{3)}$

Given the limitations of aneurysm coiling, large, giant, and fusiform aneurysms are occasionally treated with endovascular parent artery occlusion (PAO), which is divided into internal trapping and proximal occlusion. PAO is simple to perform and has superiority of a high occlusion rate and low recurrence rate; however, inherent to its deconstructive nature, PAO poses a risk of ischemic complications. ${ }^{4,5)}$

To the best of our knowledge, only several case series with a limited number of patients have reported the clinical outcomes of PAO. In this study, we aimed to elucidate the real-world outcomes of PAO performed in Japan. To achieve this goal, we retrospectively reviewed PAO data from a nationwide survey performed by the Japanese Society of Neuroendovascular Therapy (JSNET).

\section{Materials and Methods}

\section{JR-NET3 protocols}

The Japanese Registry of Neuroendovascular Therapy (JR-NET) 3 was conducted between 2010 and 2014. All the specialists certified by JSNET were instructed to register their endovascular procedures in the JR-NET 3 database. The data were collected through the JSNET coordinating office and data center, which are located at the University Medical Information Network. The institutional review boards at the participating centers approved the use of the patient data.

\section{Data extraction}

All cases of elective endovascular treatment with PAO were extracted from the JR-NET 3 database. Then, screening was performed to include only elective PAO procedures with unruptured aneurysms (i.e., ruptured aneurysms and emergent cases were excluded). Cases with incomplete data were also excluded. Ultimately, 274 cases were selected for analysis (mean patient age of $57.1 \pm 13.4$ years; $55.4 \%$ female). Figure 1 shows the data flow.

\section{Definition of complications and clinical outcomes}

Hemorrhagic complication was defined as any symptomatic intracranial hemorrhage. Ischemic complication was defined as any symptomatic intracranial ischemic event, including transient ischemic symptoms. Among ischemic complications, distal embolism was defined as any embolic infarction distal to a treated vessel, and branch occlusion was defined as the mechanical occlusion or thrombotic infarction of the territory of the branching arteries near a treated aneurysm. Any report of puncture site complication was collected, including hemorrhagic complication, ischemic complication, and infection. When worsening of modified Rankin Scale $(\mathrm{mRS}) \geq 2$ occurred, it was recorded as a "severe" complication.

In accordance with previous reports, morbidity was defined as deterioration of $\geq 2$ points on the mRS at 30 days post-intervention. ${ }^{6}$ Mortality was defined as any death within 30 days post-intervention.

\section{Statistical methods}

Variables are presented as mean \pm standard deviation (SD), count, or percentage, where appropriate. The $\chi^{2}$ test was used to compare the categorical variables, and the odds ratio (OR) was calculated for each binary variable with a 95\% confidence interval (CI). The Wilcoxon signed-rank test was used to compare the ordinal variables, while Student's all aneurysms treated with parent artery occlusion or proximal occlusion $n=1258$

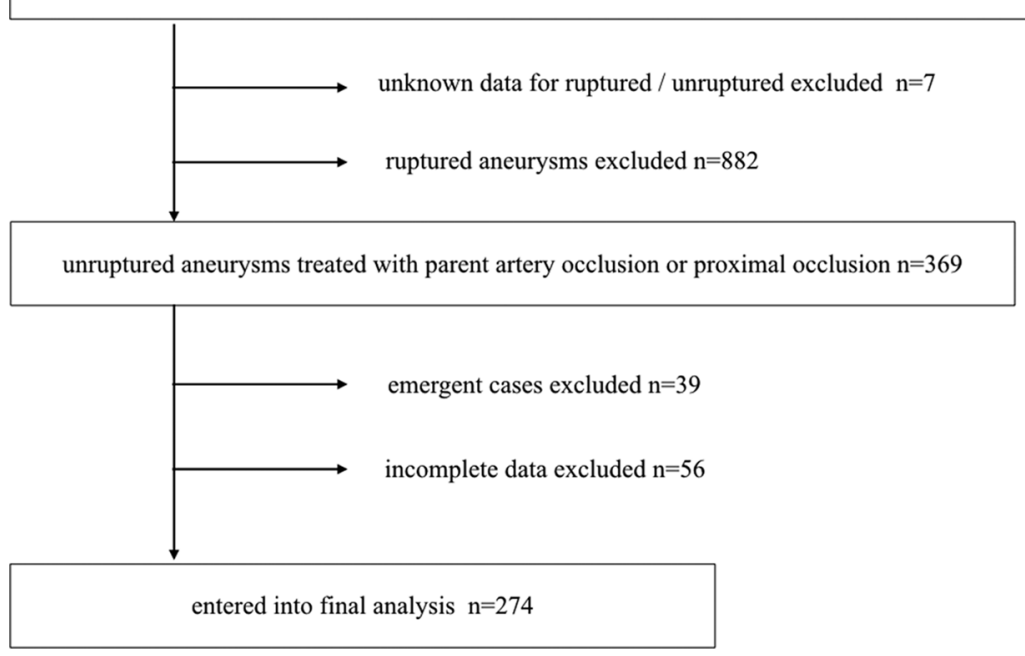

Fig. 1 Flow chart of the data in this study. There were 1258 procedures with parent artery occlusion in the JR-NET 3 database. After excluding ruptured cases and unknown causes of rupture, 369 procedures remained. Next, emergent cases and cases with incomplete data were excluded, resulting in 274 procedures being included for analysis. JR-NET: Japanese Registry of Neuroendovascular Therapy. 
$t$-test was used to compare continuous variables. To investigate the association with complications, the pretreatment variables were first analyzed using univariate analysis, and then were entered into a multiple logistic regression analysis. $P$-values $<0.05$ were considered statistically significant. If the $P$-value was less than 0.001 , it is expressed as $P<0.001$. JMP 12.2.0 software was used to perform all the analyses.

\section{Results}

\section{Aneurysm characteristics}

Table 1 summarizes the aneurysm characteristics in this study. A total of 274 cerebral aneurysms were included in this study, of which 112 (40.8\%) were located in the anterior circulation and 162 $(59.2 \%)$ were located in the posterior circulation. Among the 112 anterior circulation aneurysms, $74(66.0 \%)$ were cavernous internal carotid artery (ICA) aneurysms, 19 (16.9\%) were paraclinoid ICA

Table 1 Patient and aneurysm characteristics, $n=274$

\begin{tabular}{|c|c|}
\hline Age mean \pm SD & $57.1 \pm 13.4$ \\
\hline Female, $n(\%)$ & $155(55.4)$ \\
\hline \multicolumn{2}{|l|}{ Aneurysm location } \\
\hline Anterior circulation & $112(40.8 \%)$ \\
\hline IC-cavernous & $74(27.0 \%)$ \\
\hline IC-paraclinoid & $19(6.9 \%)$ \\
\hline IC-supraclinoid & $5(1.8 \%)$ \\
\hline Other & $15(10.1 \%)$ \\
\hline Posterior circulation & $162(59.1 \%)$ \\
\hline VA & $133(48.5 \%)$ \\
\hline BA & $5(1.8 \%)$ \\
\hline PCA & $12(4.3 \%)$ \\
\hline Other & $12(4.3 \%)$ \\
\hline \multicolumn{2}{|l|}{ Symptoms } \\
\hline Symptomatic & $136(49.6 \%)$ \\
\hline Asymptomatic & $138(50.4 \%)$ \\
\hline \multicolumn{2}{|l|}{ Aneurysm size } \\
\hline$<5 \mathrm{~mm}$ & $23(8.2 \%)$ \\
\hline $5-10 \mathrm{~mm}$ & $83(20.2 \%)$ \\
\hline $10-25 \mathrm{~mm}$ & $108(39.4 \%)$ \\
\hline$>25 \mathrm{~mm}$ & $55(20.0 \%)$ \\
\hline \multicolumn{2}{|l|}{ Morphology } \\
\hline Saccular & $149(54.4 \%)$ \\
\hline Non-saccular & $125(45.6 \%)$ \\
\hline
\end{tabular}

BA: basilar artery, IC: internal carotid artery, PCA: posterior cerebral artery, VA: vertebral artery. aneurysms, 5 (4.4\%) were supraclinoid ICA aneurysms, and $6(5.3 \%)$ were middle cerebral artery (MCA) aneurysms. ICA aneurysm location was categorized according to a previous study. ${ }^{7}$ ) Among the 162 posterior circulation aneurysms, most (133/162; $82.0 \%)$ were VA aneurysms, $12(7.4 \%)$ were PCA aneurysms, and five $(3.0 \%)$ were BA aneurysms. ICA and VA aneurysms were comprised of more than $80 \%$ of all aneurysms (Fig. 2). In terms of morphology, 149 aneurysms were saccular (54.4\%) and 125 aneurysms were non-saccular, including a fusiform, serpentine shape. There were 108 large aneurysms $(>10 \mathrm{~mm})$ and 55 giant aneurysms $(>25 \mathrm{~mm})$. Twenty-three aneurysms were small $(<5 \mathrm{~mm})$, most of which had a non-saccular shape.

\section{Treatment strategy and immediate angiographical outcomes}

For treatment strategy, internal trapping was planned for 213 cases $(77.7 \%)$ and proximal occlusion was planned for 61 cases $(22.2 \%)$ (Table 2). The rates of proximal occlusion based on aneurysm location were as follows: Cavernous ICA aneurysm, 21/74 (28.3\%); paraclinoid ICA aneurysm, 7/19 (36.8\%); supraclinoid ICA aneurysm, 1/5 (20.0\%); MCA aneurysm, 1/6 (16.6\%); VA aneurysm, 16/122 (13.1\%); VA-PICA aneurysm, 3/11 (27.2\%); BA aneurysm, 5/5 (100.0\%); and PCA aneurysm, 3/12 (25.0\%).

Both of the strategies had an extremely high success rate $(272 / 274 ; 99.2 \%)$. Only two cases of internal trapping failed.

At the end of the procedure, immediate angiographical occlusion was achieved in 248 cases (90.5\%). The rate of complete occlusion was significantly higher for internal trapping than proximal occlusion (92.9\% vs $81.9 \%$ : $P=0.009$ ).

All the PAO procedures were performed using detachable coils (there were no cases using a detachable balloon). Only bare-metal coils were used in more than half of the cases $(165 / 274 ; 60.5 \%)$, hydrogel coils were used in 64 cases $(23.3 \%)$, and bioactive coils were used in 39 cases $(14.2 \%)$. There were no apparent associations between coil characteristics and aneurysm occlusion rate or complication rate in our population.

\section{Periprocedural antithrombotic management}

Preprocedural antiplatelet therapy was performed in most cases (226/274; 82\%). Among them, preprocedural single antiplatelet therapy (SAPT) was administered in 68 cases $(24.8 \%)$, dual antiplatelet therapy (DAPT) in 150 cases $(54.7 \%)$, and triple antiplatelet therapy (TAPT) in eight cases $(2.9 \%)$. Almost all the cases were intra-procedurally heparinized (268/274 cases; $97.8 \%$ ). Postoperatively, $165 / 274$ cases (60.2\%) 


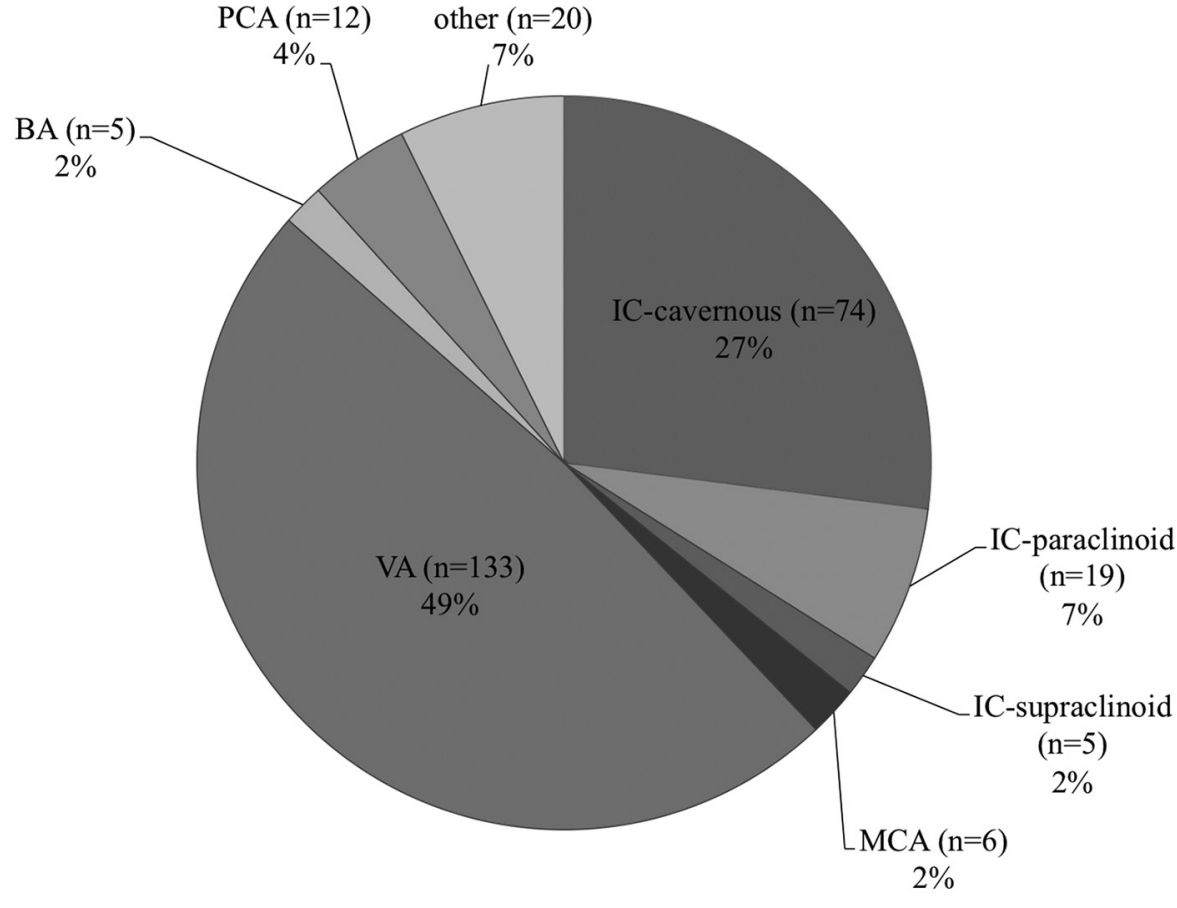

Fig. 2 Anatomical locations of the aneurysms. VA: vertebral artery, BA: basilar artery, PCA: posterior cerebral artery, IC: internal carotid artery, and MCA: middle cerebral artery.
Table 2 Procedural outcomes, complications, and clinical outcomes, $n=274$

\begin{tabular}{lc}
\hline Treatment strategy & \\
Internal trapping & $213(77.7 \%)$ \\
Proximal occlusion & $61(22.2 \%)$ \\
Technical and angiographical outcome & \\
Technical success & $272(99.7 \%)$ \\
Complete occlusion & $248(90.5 \%)$ \\
Partial occlusion & $19(6.9 \%)$ \\
Complications & \\
All complications & $60(21.8 \%)$ \\
Puncture site complications & $4(1.4 \%)$ \\
All hemorrhagic complications* & $7(2.5 \%)$ \\
Severe hemorrhagic complications** & $2(0.73 \%)$ \\
All ischemic complications*** & $48(17.5 \%)$ \\
Severe ischemic complications** & $5(1.8 \%)$ \\
Distal embolism & $22(8.0 \%)$ \\
Branch occlusion & $13(4.7 \%)$ \\
Thinical outcome & \\
\hline$*$ Ahe 30-day morbidity & $31(11.3 \%)$ \\
\hline & $2(0.7 \%)$ \\
\hline
\end{tabular}

*All symptomatic intracranial hemorrhage, ** Severe complications is defined by worsening of $\mathrm{mRS} \geq 2,{ }^{* * *}$ All symptomatic intracranial ischemic events. received anticoagulation therapy (heparin; 40 cases, argatroban; 106 cases, both heparin and argatroban; 19 cases).

Postprocedural SAPT was performed in 73 cases $(26.6 \%)$, DAPT in 148 cases $(54.0 \%)$, and TAPT in 9 cases $(3.2 \%)$. In 21 cases $(7.6 \%)$, antiplatelet therapy was strengthened after the procedure (i.e., antiplatelet drugs were added to preprocedural regimen after PAO). There were no apparent associations between any of the preprocedural or postprocedural antithrombotic therapies and ischemic complications (Table 3).

\section{Periprocedural complications}

Complications occurred in 60 cases $(21.8 \%)$, including 48 cases $(17.5 \%)$ of ischemic complications, seven cases $(2.5 \%)$ of hemorrhagic complications, and four cases $(1.4 \%)$ of puncture site complications (Table 2).

Most of the complications were ischemic complications. Of these, nearly half of the cases (20/48 cases) showed only transient symptoms, while five cases $(1.8 \%)$ were severe ischemic complications that led to morbidity. Of the ischemic complication types, there were 22 distal embolisms and 13 branch occlusions.

The rates of ischemic complications by aneurysm location were as follows: Cavernous ICA aneurysm, 13/74 (17.5\%); paraclinoid ICA aneurysm, 2/19 (10.5\%); supraclinoid ICA aneurysm, 0/5 (0.0\%); MCA aneurysm, 0/6 (0.0\%); VA aneurysm, 24/122 
Table 3 Periprocedural antithrombotic management and ischemic complications

\begin{tabular}{|c|c|c|c|c|}
\hline Management & All & $\begin{array}{c}\text { Ischemic } \\
\text { event }\end{array}$ & $\begin{array}{l}\text { No ischemic } \\
\text { event }\end{array}$ & $P$-value \\
\hline \multicolumn{5}{|c|}{ Preprocedural antiplatelet regimen } \\
\hline None & 48 & $7(14.5)$ & $41(85.4)$ & 0.83 \\
\hline $\begin{array}{l}\text { Single } \\
\text { antiplatelet } \\
\text { therapy }\end{array}$ & 68 & $11(16.1)$ & $57(83.8)$ & \\
\hline $\begin{array}{l}\text { Dual } \\
\text { antiplatelet } \\
\text { therapy }\end{array}$ & 150 & $29(19.3)$ & $121(80.6)$ & \\
\hline $\begin{array}{l}\text { Triple } \\
\text { antiplatelet } \\
\text { therapy }\end{array}$ & 8 & $1(12.5)$ & $7(87.5)$ & \\
\hline \multicolumn{5}{|c|}{ Postprocedural anticoagulation } \\
\hline None & 109 & $12(11.0)$ & $97(88.9)$ & 0.14 \\
\hline Heparin & 59 & $13(22.0)$ & $46(77.9)$ & \\
\hline Argatroban & 106 & $23(21.7)$ & $83(78.3)$ & \\
\hline \multicolumn{5}{|c|}{ Postprocedural antiplatelet regimen } \\
\hline None & 44 & $5(11.3)$ & $39(88.6)$ & 0.40 \\
\hline $\begin{array}{l}\text { Single } \\
\text { antiplatelet } \\
\text { therapy }\end{array}$ & 73 & $11(15.0)$ & $62(84.9)$ & \\
\hline $\begin{array}{l}\text { Dual } \\
\text { antiplatelet } \\
\text { therapy }\end{array}$ & 148 & $31(20.9)$ & $117(79.0)$ & \\
\hline $\begin{array}{l}\text { Triple } \\
\text { antiplatelet } \\
\text { therapy }\end{array}$ & 9 & $1(11.1)$ & $8(88.8)$ & \\
\hline
\end{tabular}

(19.6\%); VA-PICA aneurysm, 0/11 (0.0\%); BA aneurysm, 0/5 (0.0\%); and PCA aneurysm, 5/12 $(41.6 \%)$ (Fig. 3).

Hemorrhagic complications occurred in seven cases $(2.5 \%)$, two of which were severe complications $(0.7 \%)$. The details of the hemorrhagic complications are as follows: Three vessel perforations, one intra-procedural aneurysm rupture, and three cases of spontaneous intracranial hemorrhages within $24 \mathrm{~h}$ post-operation. The rates of hemorrhagic complications by aneurysm location were as follows: Cavernous ICA aneurysm, 1/74 (1.3\%); paraclinoid ICA aneurysm, 0/17 (0.0\%); supraclinoid ICA aneurysm; 0/5 $(0.0 \%)$; MCA aneurysm, 1/6 (16.6\%); VA aneurysm, 2/122 (1.6\%); VA-PICA aneurysm, 0/11 (0.0\%); BA aneurysm, 0/5 (0.0\%); and PCA aneurysm, 2/12 $(16.6 \%)$ (Fig. 3).

Among the pretreatment variables, aneurysm location and small size $(<5 \mathrm{~mm})$ were significantly associated with hemorrhagic complications according to univariate analysis. With multiple logistic regression analysis, only small size $(<5 \mathrm{~mm})$ was associated with hemorrhagic complications (OR 9.85 [1.07-221.0], $P=0.04$ ), while "patients aged 70 and older" was associated with ischemic complications (OR 2.34 [1.02-5.25], $P=0.04$ ) (Table 4). None of the pretreatment variables were associated with puncture site complications.

\section{Thirty-day clinical outcomes}

For the overall study population, the 30-day morbidity and mortality were $5.8 \%$ and $0.7 \%$, respectively (Table 2). There were two cases of mortality within

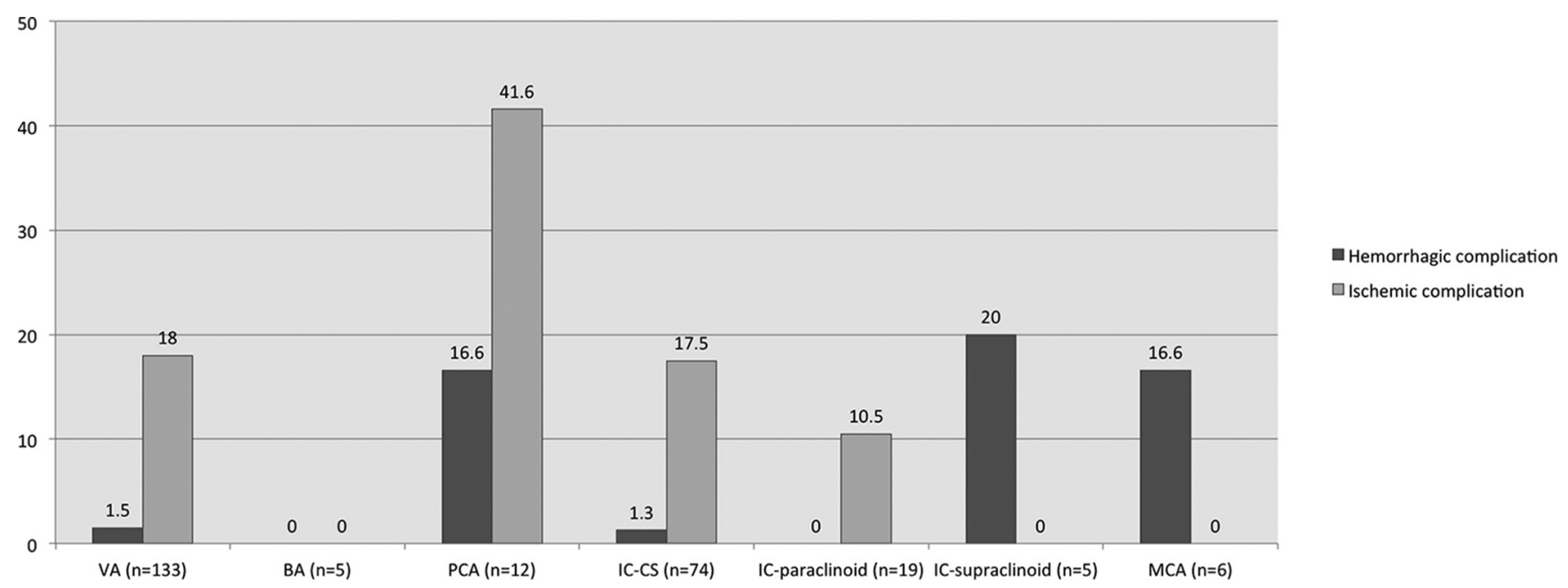

Fig. 3 Hemorrhagic and ischemic complication rates according to aneurysm location. Ischemic complications were highest for PCA aneurysms, followed by VA and IC-CS aneurysms. VA: vertebral artery, BA: basilar artery, PCA: posterior cerebral artery, IC: internal carotid artery, CS: cavernous sinus, and MCA: middle cerebral artery. 
Table 4 Risk factors for complications with PAO

\begin{tabular}{|c|c|c|c|c|}
\hline & \multicolumn{2}{|c|}{ Univariate analysis } & \multicolumn{2}{|c|}{ Multiple logistic regression } \\
\hline & OR $[95 \% \mathrm{CI}]$ & $P$-value & OR $[95 \% \mathrm{CI}]$ & $P$-value \\
\hline \multicolumn{5}{|l|}{ Hemorrhagic complications } \\
\hline Age $>70$ & 0 & 0.18 & $3.71 \times 10^{-7}(0-4.55)$ & 0.26 \\
\hline Aneurysm location & - & 0.01 & - & 0.41 \\
\hline Small aneurysms $(<5 \mathrm{~mm})$ & $17.0(3.55-81.8)$ & $<0.001$ & $9.85(1.07-221.0)$ & 0.04 \\
\hline Non-saccular shape & $1.60(0.35-7.32)$ & 0.53 & $0.99(1.07-221.0)$ & 0.99 \\
\hline Treatment strategy (IT or PO) & $1.73(0.20-14.7)$ & 0.60 & $2.01(0.18-70.6)$ & 0.59 \\
\hline $\begin{array}{l}\text { Pretreatment antiplatelet } \\
\text { therapy }(0-3)\end{array}$ & - & 0.14 & - & 0.17 \\
\hline \multicolumn{5}{|l|}{ Ischemic complications } \\
\hline Age $>70$ & $1.72(0.83-3.55)$ & 0.13 & $2.34(1.02-5.25)$ & 0.04 \\
\hline Aneurysm location & - & 0.27 & - & 0.22 \\
\hline Small aneurysms $(<5 \mathrm{~mm})$ & $1.76(0.65-4.74)$ & 0.25 & $2.56(0.70-8.74)$ & 0.14 \\
\hline Non-saccular shape & $1.18(0.59-2.08)$ & 0.72 & $1.04(0.45-2.45)$ & 0.91 \\
\hline Treatment strategy (IT or PO) & $1.10(0.51-2.37)$ & 0.79 & $0.96(0.41-2.40)$ & 0.93 \\
\hline $\begin{array}{l}\text { Pretreatment antiplatelet } \\
\text { therapy }(0-3)\end{array}$ & - & 0.83 & - & 0.73 \\
\hline
\end{tabular}

IT: internal trapping, PAO: parent artery occlusion, PO: proximal occlusion.

30 days of treatment: one case was due to intracranial hemorrhage within 24 hours post-operation for an ICA cavernous aneurysm in which case DAPT was administered preoperatively and argatroban was used postoperatively, and the other case was not related to neurological complications.

There were 16 cases of morbidity. Among these, five cases were caused by ischemic complications, one case was caused by hemorrhagic complications, one case was caused by systemic complications, and the other cases were not associated with any periprocedural complications recorded in this study. Regarding ischemic complications, three cases were caused by branch occlusion, one was caused by distal embolism, and the other was not defined. While the branch occlusion was significantly associated with morbidity $(P=0.006)$, distal embolism was not $(P=0.49)$.

\section{Discussion}

\section{Key results}

Here, we reported the current status of PAO for unruptured cerebral aneurysms in Japan.

Parent artery occlusion for unruptured cerebral aneurysms was highly feasible and had favorable angiographical results (complete occlusion was achieved in $90 \%$ of cases). The morbidity and mortality rates at 30 postoperative days were $5.8 \%$ and $0.7 \%$, respectively.
Although the rate of complications was relatively high $(21.8 \%)$, severe complications (which led to morbidity) occurred in only $2.5 \%$ of all cases. Against all expectations, an aggressive preprocedural antiplatelet regimen was not associated with reduced ischemic complications in our data; only "older age (age 70 and older)" was detected as a risk factor for ischemic complications. Hemorrhagic complications were relatively rare $(2.5 \%)$; however, one-third of hemorrhagic events led to morbidity. "Small aneurysms" were revealed as a risk factor for hemorrhagic complications.

\section{Interpretation}

This study revealed that the technical feasibility of PAO was excellent. However, the complication rate was relatively high, with $80 \%$ of complications being ischemic complications.

In previous research, the rate of ischemic complications for PAO for unruptured aneurysms was reported as $6.8-32.1 \% .^{6,8-16)}$ Thus, the rate of ischemic complications reported here is consistent with previous reports.

Since it is a deconstructive strategy, it seems natural that ischemic complications are related largely to hemodynamic disruption of the parent artery. Indeed, it has been reported that the ischemic complications in PAO are associated with hemodynamic tolerability, which is estimated by the balloon occlusion test (BOT). ${ }^{4,15,17)}$ In addition, 
extracranial-intracranial bypass can influence the risk of ischemic complications. Unfortunately, the JR-NET survey did not collect data on BOT or concomitant bypass surgery; therefore, these hemodynamic factors could not be estimated, and may bias the statistical analysis in the current study.

However, we do have detailed data on periprocedural antithrombotic therapy. Although aggressive antiplatelet therapy has been reported to reduce ischemic events in neurointervention, any kind of antiplatelet regimen was not significantly associated with ischemic complications in this study. ${ }^{18)}$ Previously, Ishii et al. ${ }^{6}$ reported details of periprocedural antithrombotic therapy with PAO from the JR-NET 1 and 2 surveys. Preprocedural DAPT was performed in $31.2 \%$ of cases in the JR-NET 1 (2005-2006) and $43.8 \%$ of cases in the JR-NET 2 (2007-2009). In this study, which is based on the JR-NET 3 (2010-2014), more than half of the cases $(54.7 \%)$ received preprocedural DAPT. Increasing number of patients are being treated with aggressive antiplatelet therapy; otherwise, the ischemic complication rate has not declined (ischemic complication rates of $12.9 \%$ in the JR-NET 1 , $12.8 \%$ in the JR-NET 2 , and $17.5 \%$ in the JR-NET 3 ). Further, no apparent association between the type of the preoperative antiplatelet regimen and the ischemic complications was noted.

It may seem that the aggressive antiplatelet therapy has limited effect on the prevention of ischemic complications with PAO; however, these results should be interpreted with caution as the ischemic complication rate may be biased by the hemodynamic factors mentioned earlier. In addition, there may be selection bias, like surgeons' choice to introduce aggressive antithrombotic therapy for aneurysms with a high risk of ischemia.

The only risk factor for ischemic complications found in this study was patient's age. Previous studies have reported that cardiovascular risk factors (such as hypertension) are a risk factor for ischemic complications with PAO. ${ }^{5,15)}$ Since the JR-NET survey did not collect data on comorbidities, we may have detected age as a confounding marker of vascular risk factors. A more detailed study is required to reveal this point.

Regarding location, PCA aneurysms had extremely high ischemic complication rates (more than $40 \%$ ). The patients with PCA aneurysms were older than the other patients [mean age (years): PCA aneurysms $57.4 \pm 3.87$; other aneurysms, $49.7 \pm 0.82$; $P=0.05]$. Apart from this, there was no statistically significant difference between PCA aneurysms and the other aneurysms in terms of morphology, treatment strategy, antithrombotic therapy, and immediate occlusion status. Previously, Matsumura et al. ${ }^{19)}$ reported a similar rate of ischemic complications with PAO for ruptured and unruptured PCA aneurysms (2/5 cases; $40 \%$ ). Xu et al. ${ }^{20)}$ also reported an extremely high rate of ischemic complications with ruptured PCA aneurysms $(8 / 8 ; 100 \%)$. These reports indicate that the fetal type PCA is a strong risk factor of ischemic complications. Although this anatomical characteristic (adult type or fetal type) was not recorded in our study, it is an important point when considering the indication of PAO for PCA aneurysms, especially unruptured cases.

Finally, these clinical results should be compared carefully with other treatment options, such as surgical clipping, surgical trapping, surgical proximal ligation, aneurysm coiling with various adjuvant techniques, and newer treatment devices. One of these new devices is the flow diverter (FD). A recent meta-analysis of 3125 patients reported that FD was associated with a $17 \%$ complication rate (ischemic complications $7.5 \%$; hemorrhagic complication $4.7 \%$ ), $4.5 \%$ neurological morbidity, and $2.8 \%$ morbidity. ${ }^{21}$

Since FD was approved in Japan in 2015, an increasing number of large and giant ICA aneurysms will be treated with FD. In the future, the clinical outcomes of PAO should be compared with those of FD.

\section{Limitations}

This study was subject to several limitations. First, this was a retrospective study of nation-wide survey data. In our analysis, 55 cases were excluded due to incomplete data, which accounted for as much as $14 \%$ of all the PAO cases for unruptured aneurysms. A relatively high amount of data exclusion can damage the reliability of the results.

Second, the JR-NET 3 registry did not collect information on BOT and extracranial-intracranial bypass surgery, patient comorbidities, or detailed anatomical characteristics (as already mentioned). These variables are reported to be associated with ischemic complications and thus may have influenced ischemic complication rates in this study.

Third, clinical outcomes were recorded at the 30 day follow-up and there is a lack of information on long-term outcomes. The clinical results of this study should be carefully compared with other reports, particularly those that assessed clinical outcomes at 90 days follow-up or longer (as there is the possibility of recovery with a longer followup time course, and the possibility of late-onset complications). 


\section{Conclusion}

We reported the current status of PAO for unruptured cerebral aneurysms in Japan. In most of the cases the PAO for unruptured cerebral aneurysms was highly feasible and achieved complete occlusion. The morbidity and mortality rates at 30 days postoperation were acceptable. However, the symptomatic ischemic complication rate of $17 \%$ was relatively high. Aggressive preprocedural antiplatelet therapy was not associated with reduced risk of ischemic complications. Given the high risk of ischemic complications, deconstructive treatment strategies should be carefully considered alongside other treatment options, like the flow diverter.

\section{Acknowledgments}

The authors would like to express their heartfelt thanks to the doctors who devoted their time to this investigation. The JR-NET3 Study Group: Co-Principle investigator; Nobuyuki Sakai, Kobe City Medical Center General Hospital, Kobe, Japan: Koji Iihara, Kyushu University, Fukuoka, Japan, Tetsu Satow, National Cerebral and Cardiovascular Center, Suita, Japan; Investigators; Masayuki Ezura, Sendai Medical Center, Sendai, Japan, Akio Hyodo, Dokkyo Medical University Saitama Medical Center, Koshigaya, Japan, Shigeru Miyachi, Aichi Medical University, Aichi, Japan, Susumu Miyamoto, Kyoto University, Kyoto, Japan, Yoji Nagai, Kobe University, Kobe, Japan, Kunihiro Nishimura, National Cerebral and Cardiovascular Center, Suita, Japan, Kazunori Toyoda, National Cerebral and Cardiovascular Center, Suita, Japan; Co-investigators; Toshiyuki Fujinaka, Osaka Medical Center, Osaka, Japan, Toshio Higashi, Fukuoka University, Fukuoka, Japan, Masaru Hirohata, Kurume University, Kurume, Japan, Japan, Akira Ishii, Kyoto University, Kyoto, Japan, Hirotoshi Imamura, Kobe City Medical Center General Hospital, Kobe, Japan, Yasushi Ito, Shinrakuen Hospital, Niigata, Japan, Naoya Kuwayama, Toyama University, Toyama, Japan, Hidenori Oishi, Juntendo University, Tokyo, Japan, Yuji Matsumaru, Tsukuba University, Tsukuba, Japan, Yasushi Matsumoto, Konan Hospital, Sendai, Japan, Ichiro Nakahara, Fujita Medical University, Aichi, Japan, Chiaki Sakai, Hyogo College of Medicine, Nishinomiya, Japan, Kenji Sugiu, Okayama University, Okayama, Japan, Tomoaki Terada, Showa University Fujigaoka Hospital, Kanagawa, Japan, Shinichi Yoshimura, Hyogo College of Medicine, Nishinomiya, Japan, and Certified Specialist of Japanese Society of Neuroendovascular Therapy.

\section{Conflicts of Interest Disclosure}

The authors declare that they have no conflicts of interest except for N. Sakai.

N. Sakai report non-related research grants from Terumo and Taiichi-Sankyo, lecturer's fees from Jimro, Otsuka, Johnson \& Johnson, Medtronic, Stryker, Terumo and Medico's Hirata; membership on the advisory boards for Medtronic and Jimro.

All the authors who are members of the Japan Neurosurgical Society (JNS) have registered selfreported conflict of interest disclosure statement forms online through the website for JNS members.

\section{References}

1) Molyneux A, Kerr R, Stratton I, et al.; International Subarachnoid Aneurysm Trial (ISAT) Collaborative Group: International Subarachnoid Aneurysm Trial (ISAT) of neurosurgical clipping versus endovascular coiling in 2143 patients with ruptured intracranial aneurysms: a randomized trial. Lancet 360: 1267-1274, 2002

2) Sluzewski M, Menovsky T, van Rooij WJ, Wijnalda D: Coiling of very large or giant cerebral aneurysms: long-term clinical and serial angiographic results. AJNR Am J Neuroradiol 24: 257-262, 2003

3) Piotin M, Blanc R, Spelle L, et al.: Stent-assisted coiling of intracranial aneurysms: clinical and angiographic results in 216 consecutive aneurysms. Stroke 41: 110-115, 2010

4) Clarençon F, Bonneville F, Boch AL, Lejean L, Biondi A: Parent artery occlusion is not obsolete in giant aneurysms of the ICA. Experience with very-long-term follow-up. Neuroradiology 53: 973-982, 2011

5) Labeyrie MA, Lenck S, Bresson D, et al.: Parent artery occlusion in large, giant, or fusiform aneurysms of the carotid siphon: clinical and imaging results. AJNR Am J Neuroradiol 36: 140-145, 2015

6) Ishii A, Miyamoto S, Ito Y, Fujinaka T, Sakai C, Sakai N; Japanese Registry of Neuroendovascular Therapy Investigators: Parent artery occlusion for unruptured cerebral aneurysms: the Japanese Registry of Neuroendovascular Therapy (JR-NET) 1 and 2. Neurol Med Chir (Tokyo) 54: 91-97, 2014

7) Iihara K, Murao K, Sakai N, et al.: Unruptured paraclinoid aneurysms: a management strategy. J Neurosurg 99: 241-247, 2003

8) Berenstein A, Ransohoff J, Kupersmith M, Flamm E, Graeb D: Transvascular treatment of giant aneurysms of the cavernous carotid and vertebral arteries: functional investigation and embolization. Surg Neurol 21: 3-12, 1984

9) Bechan RS, Majoie CB, Sprengers ME, Peluso JP, Sluzewski M, van Rooij WJ: Therapeutic internal carotid artery occlusion for large and giant 
aneurysms: a single center cohort of 146 patients. AJNR Am J Neuroradiol 37: 125-129, 2016

10) Larson JJ, Tew JM Jr, Tomsick TA, van Loveren HR: Treatment of aneurysms of the internal carotid artery by intravascular balloon occlusion: long-term followup of 58 patients. Neurosurgery 36: 26-30, 1995

11) Bavinzski G, Killer M, Ferraz-Leite H, Gruber A, Gross CE, Richling B: Endovascular therapy of idiopathic cavernous aneurysms over 11 years. AJNR Am J Neuroradiol 19: 559-565, 1998

12) Murakami K, Shimizu H, Matsumoto Y, Tominaga T: Acute ischemic complications after therapeutic parent artery occlusion with revascularization for complex internal carotid artery aneurysms. Surg Neurol 71: 434-441, 2009

13) Shimizu H, Matsumoto Y, Tominaga T: Parent artery occlusion with bypass surgery for the treatment of internal carotid artery aneurysms: clinical and hemodynamic results. Clin Neurol Neurosurg 112: 32-39, 2010

14) Zanaty M, Chalouhi N, Starke RM, et al.: Flow diversion versus conventional treatment for carotid cavernous aneurysms. Stroke 45: 2656-2661, 2014

15) Shimizu K, Imamura H, Mineharu Y, et al.: Endovascular parent-artery occlusion of large or giant unruptured internal carotid artery aneurysms. A long-term single-center experience. J Clin Neurosci 37: 73-78, 2017

16) Kashiwazaki D, Ushikoshi S, Asano T, Kuroda S, Houkin K: Long-term clinical and radiological results of endovascular internal trapping in vertebral artery dissection. Neuroradiology 55: 201-206, 2013
17) van Rooij WJ, Sluzewski M, Slob MJ, Rinkel GJ: Predictive value of angiographic testing for tolerance to therapeutic occlusion of the carotid artery. AJNR Am J Neuroradiol 26: 175-178, 2005

18) Enomoto Y, Yoshimura S, Sakai N, Egashira Y; Japanese Registry of Neuroendovascular Therapy (JR-NET) investigators: Current perioperative management of anticoagulant and antiplatelet use in neuroendovascular therapy: analysis of JR-NET 1 and 2. Neurol Med Chir (Tokyo) 54 Suppl 2: 9-16, 2014

19) Matsumura H, Kato N, Fujiwara Y, et al.: Endovascular treatments for posterior cerebral artery aneurysms and vascular insufficiency of fetal-type circulation after parent artery occlusion. J Clin Neurosci 32: 41-46, 2016

20) Xu J, Xu L, Wu Z, Chen X, Yu J, Zhang J: Fetal-type posterior cerebral artery: the pitfall of parent artery occlusion for ruptured $\mathrm{P}_{2}$ segment and distal aneurysms. J Neurosurg 123: 906-914, 2015

21) Zhou G, Su M, Yin YL, Li MH: Complications associated with the use of flow-diverting devices for cerebral aneurysms: a systematic review and meta-analysis. Neurosurg Focus 42: E17, 2017

Address reprint requests to: Akira Ishii, $\mathrm{MD}, \mathrm{PhD}$, Department of Neurosurgery, Graduate School of Medicine, Kyoto University, 54 Shogoin Kawahara-cho, Sakyo-ku, Kyoto, Kyoto 606-8397, Japan. e-mail: ishii@kuhp.kyoto-u.ac.jp 